\title{
NONCOMMUTATIVE ERGODIC AVERAGES OF BALLS AND SPHERES OVER EUCLIDEAN SPACES
}

\author{
GUIXIANG HONG
}

\begin{abstract}
In this paper, we establish a noncommutative analogue of Calderón's transference principle, which allows us to deduce noncommutative ergodic maximal inequalities from the special case- operatorvalued maximal inequalities. As applications, we deduce dimensionfree estimates of noncommutative Wiener's maximal ergodic inequality and noncommutative Stein-Calderón's maximal ergodic inequality over Euclidean spaces. We also show the corresponding individual ergodic theorems. To show Wiener's pointwise ergodic theorem, we construct a dense subset on which pointwise convergence holds following a somewhat standard way. To show Jones' pointwise ergodic theorem, we use again transference principle together with Littlewood-Paley method, which is different from Jones' original variational method that is still unavailable in the noncommutative setting.
\end{abstract}

\section{INTRODUCTION}

Let $\beta_{r}$ (resp. $\sigma_{r}$ ) be the normalized Lebesgue measure on the Euclidean ball $\left\{v \in \mathbb{R}^{n} ;|v|_{\ell_{2}} \leq r\right\}$ (resp. the Euclidean sphere $\left\{v \in \mathbb{R}^{n}:|v|_{\ell_{2}}=\right.$ $r\})$. Let $(X, m)$ be a standard measure space on which $\mathbb{R}^{n}$ acts measurably by measure preserving transformation $\pi$. Let $\pi\left(\beta_{r}\right)$ (resp. $\pi\left(\sigma_{r}\right)$ ) denotes the operator canonically associated to $\beta_{r}$ (resp. $\sigma_{r}$ ) on $L_{p}(X)$. Wiener's pointwise ergodic theorem [32] asserts that $\pi\left(\beta_{r}\right) f(x)$ convergence to a limit as $r \rightarrow \infty$ for almost every $x \in X$ provided $f \in L_{p}(X)$ with $1 \leq p<\infty$. The limit is given by $F(f)$, the projection of $f$ to the fixed point subspace $\left\{g \in L_{p}(X): \pi(v) g=g, \forall v \in \mathbb{R}^{n}\right\}$. While Jones' pointwise ergodic theorem [19] asserts $\pi\left(\sigma_{r}\right) f(x)$ convergence to $F(f)$ as $r \rightarrow \infty$ for almost every $x \in X$ provided $f \in L_{p}(X)$ with $n /(n-1)<p<\infty$ and $n>2$ (See [21] for the case $n=2$ ).

The main tool used in the proof of pointwise convergence is maximal inequality. In both the ball and sphere cases, the ergodic maximal inequalities

MR(2010) Subject Classification. Primary 46L52, 37A15; Secondary 46L51, $42 \mathrm{~B} 25$.

Keywords. Noncommutative $L_{p}$ space, transference principle, ball and sphere averages, maximal ergodic theorem, individual ergodic theorem, spectral method. 
are deduced, through Calderón's transference principle, from the corresponding maximal inequalities in the case $X=\mathbb{R}^{n}$ and the action given by translation, that is, from the Hardy-Littlewood maximal inequality (in the ball case) and Stein's spherical maximal inequality (in the sphere case). Once the ergodic maximal inequality is available, to show the individual ergodic theorem it suffices to identify a dense subset on which pointwise convergence holds. That the method in constructing a dense subset in the ball case is now standard. However, it is usually a difficult task to identify a dense subset in the sphere case since the spherical measure is singular. See Jones' original proof [19].

The main purpose of this paper is to establish Wiener's and Jones' results in the noncommutative setting. That means, we are going to build maximal ergodic theorems and then pointwise ergodic theorems for general $W^{*}$-dynamical system $\left(\mathcal{M}, \tau, \mathbb{R}^{n}, \alpha\right)$, where $(\mathcal{M}, \tau)$ is a von Neumann algebra equipped with a trace $\tau$ and $\alpha: \mathbb{R}^{n} \rightarrow \operatorname{Aut}(\mathcal{M})$ is a continuous trace-preserving group homomorphism (also called an action) in the weak *-topology. If we take for $\mathcal{M}$ the algebra $L_{\infty}(X, m)$ with $(X, m)$ a measure space, $\tau$ the associated integral and $\alpha$ induced by an invertible measurepreserving transformation of $X$, we will recover Wiener's and Jones' results.

For the main purpose, we first establish a noncommutative version of Calderón's transference principle. Calderón's original arguments do not work in the present setting, since there does not exist perfect notion of "point" on the noncommutative measure spaces. We overcome this difficulty using the ideas developed in the theory of vector-valued noncommutative $L_{p}$ spaces. The noncommutative transference principle reduce maximal ergodic inequalities to the operator-valued maximal inequalities, which have been shown in [23], [12]. It is worth to mention that in [23], Mei used noncommutative Doob's inequality [15] to prove the Hardy-Littlewood maximal inequality on $\mathbb{R}^{n}$, which yields that the bounds are of order $O\left(2^{n}\right)$. While in [12], the author show that the bounds could be taken to be independent of $n$ by adapting Stein-Strömberg's idea [29], which is interesting in its own right.

As in the classical setting, with maximal inequality at hand, to use density arguments to show the pointwise convergence, it suffices to find some dense subset such that pointwise convergence holds on it. In Wiener's case, we construct a dense subset following a somewhat standard way, see Section 4 below. However, since sphere measures are singular, the dense subset contructed previously does not work in Jones' case. On the other hand, Jones' original variational method remains an open problem in the noncommutative setting. Our first attempt is via spectral method. We construct dense subsets on which pointwise convergence holds only when the dimension of Euclidean spaces $n \geq 4$, see Remark 6.4. Motivated by Rubio de Francia's 
proof of Stein's spherical maximal inequality (see for instance [26] [14]), we use Littlewood-Paley function to decompose spherical means into pieces, and use transference principle to show each piece satisfies a generalized noncommutative Wiener's ergodic theorem. Summing up all the pieces, we obtain noncommutative Jones' ergodic theorem for all $n \geq 3$, see Section 5 below.

As it is well-known that in the classical setting, Wiener's ergodic theorem inspires many mathematicians to study the ball and sphere averaging problems in ergodic theory associated to more general groups, see for instance the survey paper by Nevo [24]. We expect similar story would take place in the noncommutative setting. Actually, noncommutative ergodic theory has been developed since the very beginning of the theory of "rings of operators". However at the early stage, only mean ergodic theorems have been obtained. It is until 1976 after Lance's pioneer work [20] that the study of individual ergodic theorems really took off. Lance proved that the ergodic averages associated with an automorphism of a $\sigma$-finite von Neumann algebra which leaves invariant a normal faithful state converge almost uniformly. Lance's work motivated some mathematicians to study individual ergodic theorems associated to general groups (see for instance [18] and references therein). All these results can be regarded as individual ergodic theorems in the case $p=\infty$, where maximal ergodic theorems hold trivially. On the other hand, Yeadon [33] obtained a maximal and pointwise ergodic theorem in the preduals of semifinite von Neumann algebras. Yeadon's theorem provides a maximal ergodic inequality which might be understood as a weak type $(1,1)$ inequality. In contrast with the classical theory, the noncommutative nature of these weak type $(1,1)$ inequalities seems a priori unsuitable for classical interpolation arguments. The breakthrough was made in the previously quoted paper [18] by Junge and $\mathrm{Xu}$. They established a sophisticated real interpolation method using wellestablished noncommutative $L_{p}$ theory, which together with Yeadon's weak type $(1,1)$ inequality allows them to obtain the noncommutative DunfordSchwartz maximal ergodic theorem, thus the noncommutative individual ergodic theorem in $L_{p}$ spaces for all $1 \leq p<\infty$. This breakthrough motivates further reserach on noncommutative ergodic theorems including the present paper, see also [1] [13] [2] [10] [22] [11] and references therein.

This paper is organized as follows. In the next section, we give some necessary preliminaries for formulating noncommutative ergodic theorems from the pointview of classical ergodic theory, such as noncommutative analogues of $L_{p}$ spaces, maximal norms, pointwise convergence and measure-preserving dynamical systems. In Section 3, we prove a noncommutative version of Calderón's transference principle. The noncommutative version of Wiener's ergodic theorem is shown in Section 4. Section 5 is devoted to the proof of noncommutative Jones' ergodic theorem. In the Appendix, we present the 
spectral method to find a dense subset, which is particularly useful when the underlying group is not amenable.

\section{Preliminaries AND FrameWork}

2.1. Noncommutative $L_{p}$-spaces. Let $(\mathcal{M}, \tau)$ be a noncommutative measure spaces, that is, $\mathcal{M}$ is a von Neumann algebra and $\tau$ is a normal semifinite faithful trace. Let $S_{\mathcal{M}}^{+}$be the set of all positive element $x$ in $\mathcal{M}$ with $\tau(s(x))<\infty$, where $s(x)$ is the smallest projection $e$ such that exe $=x$. Let $S_{\mathcal{M}}$ be the linear span of $S_{\mathcal{M}}^{+}$. Then any $x \in S_{\mathcal{M}}$ has finite trace, and $S_{\mathcal{M}}$ is a $w^{*}$-dense $*$-subalgebra of $\mathcal{M}$.

Let $1 \leq p<\infty$. For any $x \in S_{\mathcal{M}}$, the operator $|x|^{p}$ belongs to $S_{\mathcal{M}}^{+}$ $\left(|x|=\left(x^{*} x\right)^{\frac{1}{2}}\right)$. We define

$$
\|x\|_{p}=\left(\tau\left(|x|^{p}\right)\right)^{\frac{1}{p}}, \quad \forall x \in S_{\mathcal{M}} .
$$

One can check that $\|\cdot\|_{p}$ is well defined and is a norm on $S_{\mathcal{M}}$. The completion of $\left(S_{\mathcal{M}},\|\cdot\|_{p}\right)$ is denoted by $L_{p}(\mathcal{M})$ which is the usual noncommutative $L_{p^{-}}$ space associated with $(\mathcal{M}, \tau)$. For convenience, we usually set $L_{\infty}(\mathcal{M})=\mathcal{M}$ equipped with the operator norm $\|\cdot\|_{\mathcal{M}}$. We refer the reader to [31] for more information on noncommutative $L_{p}$-spaces.

2.2. Noncommutative maximal norms. Let us recall the definition of the noncommutative maximal norm introduced by Pisier [30] and Junge [15]. We define $L_{p}\left(\mathcal{M} ; \ell_{\infty}\right)$ to be the space of all sequences $x=\left(x_{n}\right)_{n \geq 1}$ in $L_{p}(\mathcal{M})$ which admits a factorization of the following form: there exist $a, b \in L_{2 p}(\mathcal{M})$ and a bounded sequence $y=\left(y_{n}\right)$ in $L_{\infty}(\mathcal{M})$ such that

$$
x_{n}=a y_{n} b, \quad \forall n \geq 1 .
$$

The norm of $x$ in $L_{p}\left(\mathcal{M} ; \ell_{\infty}\right)$ is given by

$$
\|x\|_{L_{p}\left(\ell_{\infty}\right)}=\inf \left\{\|a\|_{2 p} \sup _{n \geq 1}\left\|y_{n}\right\|_{\infty}\|b\|_{2 p}\right\}
$$

where the infimum runs over all factorizations of $x$ as above. We will follow the convention adopted in [18] that $\|x\|_{L_{p}\left(\ell_{\infty}\right)}$ is sometimes denoted by $\left\|\sup _{n}^{+} x_{n}\right\|_{p}$.

More generally, if $\Lambda$ is any index set, we define $L_{p}\left(\mathcal{M} ; \ell_{\infty}(\Lambda)\right)$ as the space of all $x=\left(x_{\lambda}\right)_{\lambda \in \Lambda}$ in $L_{p}(\mathcal{M})$ that can be factorized as

$$
x_{\lambda}=a y_{\lambda} b \quad \text { with } \quad a, b \in L_{2 p}(\mathcal{M}), y_{\lambda} \in L_{\infty}(\mathcal{M}), \sup _{\lambda}\left\|y_{\lambda}\right\|_{\infty}<\infty .
$$

The norm of $L_{p}\left(\mathcal{M} ; \ell_{\infty}(\Lambda)\right)$ is defined by

$$
\left\|\sup _{\lambda \in \Lambda}{ }^{+} x_{\lambda}\right\|_{p}=\inf _{x_{\lambda}=a y_{\lambda} b}\left\{\|a\|_{2 p} \sup _{\lambda \in \Lambda}\left\|y_{\lambda}\right\|_{\infty}\|b\|_{2 p}\right\} .
$$


It is shown in [18] that $x \in L_{p}\left(\mathcal{M} ; \ell_{\infty}(\Lambda)\right)$ if and only if

$$
\sup \left\{\left\|\sup _{\lambda \in J}^{+} x_{\lambda}\right\|_{p}: J \subset \Lambda, J \text { finite }\right\}<\infty \text {. }
$$

In this case, $\left\|\sup _{\lambda \in \Lambda}{ }^{+} x_{\lambda}\right\|_{p}$ is equal to the above supremum. In the following, we omit the index set $\Lambda$ when it will not cause confusion.

A closely related operator space is $L_{p}\left(\mathcal{M} ; \ell_{\infty}^{c}\right)$ for $p \geq 2$ which is the set of all sequences $\left(x_{n}\right)_{n} \subset L_{p}(\mathcal{M})$ such that

$$
\left\|\sup _{n \geq 1}^{+}\left|x_{n}\right|^{2}\right\|_{p / 2}^{1 / 2}<\infty
$$

While $L_{p}\left(\mathcal{M} ; \ell_{\infty}^{r}\right)$ for $p \geq 2$ is the Banach space of all sequences $\left(x_{n}\right)_{n} \subset$ $L_{p}(\mathcal{M})$ such that $\left(x_{n}^{*}\right)_{n} \in L_{p}\left(\mathcal{M} ; \ell_{\infty}^{c}\right)$. All these spaces fall into the scope of amalgamated $L_{p}$ spaces intensively studied in [17]. The following interpolation relationship between symmetric and asymmetric maximal norms will allow the usage of square functions to control maximal norms in Section 5.

Lemma 2.1. Let $2 \leq p \leq \infty$. Then

$$
\left(L_{p}\left(\mathcal{M} ; \ell_{\infty}^{c}\right), L_{p}\left(\mathcal{M} ; \ell_{\infty}^{r}\right)\right)_{\frac{1}{2}}=L_{p}\left(\mathcal{M} ; \ell_{\infty}\right)
$$

In addition to the strong maximal norms which correspond to $L_{p}$-norms of maximal function, we are also concerned with the weak maximal norms which correspond to weak $L_{p}$-norms of maximal function. Given a sequence $\left(x_{n}\right)_{n}$ in $L_{p}(\mathcal{M})$, we define

$$
\left\|\left(x_{n}\right)_{n}\right\|_{\Lambda_{p}\left(\ell_{\infty}\right)}=\sup _{\lambda} \lambda \inf _{e \in \mathcal{P}(\mathcal{M})}\left\{\left(\tau\left(e^{\perp}\right)\right)^{\frac{1}{p}}:\left\|e x_{n} e\right\|_{\infty} \leq \lambda\right\} .
$$

When $\mathcal{M}$ is commutative, then infimum in this definition is attained at the projection $\mathbb{1}_{[0, \lambda]}\left(\sup _{n}\left|x_{n}\right|\right)$. Thus $\Lambda_{p}\left(\ell_{\infty}\right)$-quasi norm is exactly the weak $L_{p}$-quasi norm of maximal function. If we define the quasi Banach space $\Lambda_{p}\left(\mathcal{M} ; \ell_{\infty}\right)$ to be the set of all sequence $\left(x_{n}\right)_{n} \in L_{p}(\mathcal{M})$ such that its $\Lambda_{p}\left(\ell_{\infty}\right)$ quasi norm being finite, then these spaces have some nice interpolation properties. We refer the readers to [18] for more information.

For general index set, $\Lambda_{p}\left(\ell_{\infty}\right)$-quasi norm and whence quasi Banach spaces can be defined similarly as in the definition of strong maximal norms.

2.3. Noncommutative pointwise convergence. We recall an appropriate substitute for the usual almost everywhere convergence in the noncommutative setting. This is the almost uniform convergence introduced by Lance [20] (see also [18]).

Let $\left(x_{\lambda}\right)_{\lambda \in \Lambda}$ be a family of elements in $L_{p}(\mathcal{M})$. Recall that $\left(x_{\lambda}\right)_{\lambda \in \Lambda}$ is said to converge almost uniformly to $x$, abbreviated by $x_{\lambda} \rightarrow x, a . u$ if for every $\varepsilon>0$ there exists a projection $e \in \mathcal{M}$ such that

$$
\tau(1-e)<\varepsilon \text { and } \lim _{\lambda}\left\|e\left(x_{\lambda}-x\right)\right\|_{\infty}=0 .
$$


Also, $\left(x_{\lambda}\right)_{\lambda \in \Lambda}$ is said to converge bilaterally almost uniformly to $x$, abbreviated by $x_{\lambda} \rightarrow x$, b.a.u if for every $\varepsilon>0$ there is a projection $e \in \mathcal{M}$ such that

$$
\tau(1-e)<\varepsilon \quad \text { and } \quad \lim _{\lambda}\left\|e\left(x_{\lambda}-x\right) e\right\|_{\infty}=0 .
$$

Obviously, if $x_{\lambda} \rightarrow x, a . u$, then $x_{\lambda} \rightarrow x$,b.a.u. On the other hand, in the commutative case, these two convergences are equivalent to the usual almost everywhere convergence in terms of Egorov's theorem. However they are different in the noncommutative setting.

As in [18], in order to deduce the pointwise convergence theorems from the corresponding maximal inequalities, it is convenient to use the closed subspace $L_{p}\left(\mathcal{M} ; c_{0}\right)$ of $L_{p}\left(\mathcal{M} ; \ell_{\infty}\right)$. Recall that $L_{p}\left(\mathcal{M} ; c_{0}\right)$ is defined as the space of all sequences $\left(x_{n}\right) \in L_{p}(\mathcal{M})$ such that there are $a, b \in L_{2 p}(\mathcal{M})$ and $\left(y_{n}\right) \subset \mathcal{M}$ verifying

$$
x_{n}=a y_{n} b \quad \text { and } \quad \lim _{n}\left\|y_{n}\right\|_{\infty}=0 .
$$

Similarly, for the study of the $a . u$ convergence, we use the closed subspace $L_{p}\left(\mathcal{M} ; c_{0}^{c}\right)$ of $L_{p}\left(\mathcal{M} ; \ell_{\infty}^{c}\right)$, which is defined to be the space of all sequences $\left(x_{n}\right) \in L_{p}(\mathcal{M})$ such that there are $b \in L_{p}(\mathcal{M})$ and $\left(y_{n}\right) \subset \mathcal{M}$ verifying

$$
x_{n}=y_{n} b \quad \text { and } \quad \lim _{n}\left\|y_{n}\right\|_{\infty}=0 .
$$

The following lemma will be useful for our study of individual erogdic theorem (see [6]).

Lemma 2.2. (i) If $1 \leq p<\infty$ and $\left(x_{n}\right) \in L_{p}\left(\mathcal{M} ; c_{0}\right)$, then $x_{n} \rightarrow 0$, b.a.u. (ii) If $2 \leq p<\infty$ and $\left(x_{n}\right) \in L_{p}\left(\mathcal{M} ; c_{0}^{c}\right)$, then $x_{n} \rightarrow 0$, a.u.

2.4. Group actions and related notions. We will call $\left(\mathcal{M}, \tau, \mathbb{R}^{n}, \alpha\right)$ a $W^{*}$ dynamical system if $(\mathcal{M}, \tau)$ is a noncommutative measure space and $\alpha: \mathbb{R}^{n} \rightarrow$ $\operatorname{Aut}(\mathcal{M})$ is a continuous trace-preserving group homomorphism (also called an action) in the weak $*$-topology. It is well-known that $\alpha$ is naturally extended to be isometric automorphisms of $L_{p}(\mathcal{M})$ for all $1 \leq p \leq \infty$, which is still denoted by $\alpha$. As it is well-known, the weak $*$-continuity of $(\alpha(u))_{u \in \mathbb{R}^{n}}$ on $\mathcal{M}$ induces the strong continuity of $(\alpha(u))_{u \in \mathbb{R}^{n}}$, i.e. for each $x \in L_{p}(\mathcal{M})$ with $1 \leq p<\infty$, the map $u \rightarrow \alpha(u) x$ is a continuous map from $\mathbb{R}^{n}$ to $L_{p}(\mathcal{M})$, where we take the norm topology on $L_{p}(\mathcal{M})$.

Let $\mathcal{F}=\left\{x \in \mathcal{M}: \alpha(u) x=x, \forall u \in \mathbb{R}^{n}\right\}$. It is easy to show that $\mathcal{F}$ is a von Neumann subalgebra of $\mathcal{M}$ as in the semigroup case [18], thus there exists a unique conditional expectation $F: \mathcal{M} \rightarrow \mathcal{F}$. Moreover, this conditional expectation extends naturally from $L_{p}(\mathcal{M})$ to $L_{p}(\mathcal{F})$ with $1 \leq p<\infty$, which are still denoted by $F$.

Let $M\left(\mathbb{R}^{n}\right)$ denote the Banach space of bounded complex Borel measures on $\mathbb{R}^{n}$. For each $\mu \in M\left(\mathbb{R}^{n}\right)$, there corresponds an operator $\alpha(\mu)$, with norm 
bounded by $\|\mu\|_{1}$ in every $L_{p}(\mathcal{M}), 1 \leq p \leq \infty$, given by

$$
\alpha(\mu) x=\int_{\mathbb{R}^{n}} \alpha(v) x d \mu(v), \forall x \in L_{p}(\mathcal{M}) .
$$

This definition should be justified as follows. For any $x \in L_{p}(\mathcal{M})$ and $y \in$ $L_{q}(\mathcal{M})$ where $1 / p+1 / q=1$, the function $v \rightarrow\langle\alpha(v) x, y\rangle$ is continuous on $\mathbb{R}^{n}$, bounded by $\|x\|_{p}\|y\|_{q}$. Hence

$$
\int_{\mathbb{R}^{n}}\langle\alpha(v) x, y\rangle d \mu(v) \leq\|\mu\|_{1}\|x\|_{p}\|y\|_{q} .
$$

It follows that the operator $\alpha(\mu) x$ is well defined and $\alpha(\mu) x$ is in $L_{p}(\mathcal{M})$. Moreover, $\|\alpha(\mu) x\|_{p} \leq\|\mu\|_{1}\|x\|_{p}$ so that $\|\alpha(\mu)\| \leq\|\mu\|_{1}$.

It can be easily checked that tha map $\mu \rightarrow \alpha(\mu)$ is a norm-continuous *-representation of the involutive Banach algebra $M\left(\mathbb{R}^{n}\right)$ as an algebra of operators on $L_{2}(\mathcal{M})$. We recall that the product in $M\left(\mathbb{R}^{n}\right)$ is defined as convolution $\mu * \nu(f)=\int_{\mathbb{R}^{n}} \int_{\mathbb{R}^{n}} f(u v) d \mu(u) d \nu(v)$, and the involution is $\mu^{*}(E)=$ $\overline{\mu(E)^{-1}}$.

Denote by $P\left(\mathbb{R}^{n}\right)$ the subset of probability measures in $M\left(\mathbb{R}^{n}\right)$. Let $t \rightarrow$ $\nu_{t}$ be a weakly continuous map from $\mathbb{R}_{+}$to $P\left(\mathbb{R}^{n}\right)$, namely $t \rightarrow \nu_{t}(f)$ is continuous for each $f \in C_{c}\left(\mathbb{R}^{n}\right)$. We will refer to $\left(\nu_{t}\right)_{t>0}$ as a one-parameter family of probability measures. We can now formulate the following definition.

Definition 2.3. A one-parameter family $\left(\nu_{t}\right)_{t>0} \subset P\left(\mathbb{R}^{n}\right)$ will be called a global (resp. local) noncommutative pointwise ergodic family in $L_{p}$ if for every $W^{*}$-dynamical system $\left(\mathcal{M}, \tau, \mathbb{R}^{n}, \alpha\right)$ and every $x \in L_{p}(\mathcal{M}), \alpha\left(\nu_{t}\right) x$ converge bilaterally almost uniformly to $F(x)$ (resp. $x$ ) as $t$ tends to $\infty$ (resp. 0 ).

\section{Noncommutative Calderón's transference PRinciple}

In this section, we establish a noncommutative analogue of Calderón's transference principle. It is worth to mention that all the assertions in this section are still true for general amenable groups, and thus a general version of noncommutative Calderón's transference principle is still available. We prefer to prove them rigorously in our another work, since they are not raleted to the later applications in the present paper.

Let $\lambda$ be the action of $\mathbb{R}^{n}$ on the group itself by translation. Recall that an operator $T$ is completely bounded on $L_{p}\left(\mathbb{R}^{n}\right)$ if for any noncommutative measure space $(\mathcal{N}, t r), T \otimes i d_{\mathcal{N}}$ is bounded on $L_{p}\left(L_{\infty}\left(\mathbb{R}^{n}\right) \bar{\otimes} \mathcal{N}\right)$; similar statements hold for a sequence of operators or completely weakly bounded. See [30] for detailed informations.

Theorem 3.1. Let $\left(\nu_{t}\right)_{t>0}$ be a family of probability measures on $\mathbb{R}^{n}$ having their support contained in a Euclidean ball of radius $C t$ where $C$ is an absolute constant. If the family of operators $\left(\lambda\left(\nu_{t}\right)\right)_{t>0}$ were completely bounded from 
$L_{p}\left(\mathbb{R}^{n}\right)$ to $L_{p}\left(L_{\infty}\left(\mathbb{R}^{n}\right) ; \ell_{\infty}\right)$ (resp. to $\Lambda_{p}\left(L_{\infty}\left(\mathbb{R}^{n}\right) ; \ell_{\infty}\right)$ ), then for any $W^{*}$ dynamical system $\left(\mathcal{M}, \tau, \mathbb{R}^{n}, \alpha\right)$, the family of operators $\left(\alpha\left(\nu_{t}\right)\right)_{t>0}$ is completely bounded from $L_{p}(\mathcal{M})$ to $L_{p}\left(\mathcal{M} ; \ell_{\infty}\right)$ (resp. to $\left.\Lambda_{p}\left(\mathcal{M} ; \ell_{\infty}\right)\right)$.

In the rest of this section, we fix a $W^{*}$-dynamical system $\left(\mathcal{M}, \tau, \mathbb{R}^{n}, \alpha\right)$. The following three lemmas are well-known in the commutative case, but not obvious in the noncommutative setting.

Lemma 3.2. Let $1 \leq p \leq \infty$. Then for $\left(x_{t}\right)_{t>0}$ in $L_{p}(\mathcal{M})$, we have

$$
\left\|\left(x_{t}\right)_{t>0}\right\|_{L_{p}\left(\ell_{\infty}\right)}=\sup _{T}\left\|\left(x_{t}\right)_{0<t \leq T}\right\|_{L_{p}\left(\ell_{\infty}\right)}
$$

and further we have

$$
\left\|\left(x_{t}\right)_{t>0}\right\|_{\Lambda_{p}\left(\ell_{\infty}\right)} \simeq \sup _{T}\left\|\left(x_{t}\right)_{0<t \leq T}\right\|_{\Lambda_{p}\left(\ell_{\infty}\right)}
$$

for positive $x_{t}$ 's.

Proof. The first equation has been proved in [18] using the duality between $L_{p^{\prime}}\left(\mathcal{M} ; \ell_{1}\right)$ and $L_{p}\left(\mathcal{M} ; \ell_{\infty}\right)$. Let us prove the second equivalence. The left hand side controls trivially the right hand side. Non-trivial part is the reverse inequality. We start with the observation

$$
\begin{aligned}
\left\{e \in \mathcal{P}(\mathcal{M}):\left\|e x_{t} e\right\|_{\infty} \leq \lambda, \forall t>0\right\} \\
\quad=\bigcap_{T}\left\{e \in \mathcal{P}(\mathcal{M}):\left\|e x_{t} e\right\|_{\infty} \leq \lambda, \forall 0<t \leq T\right\} .
\end{aligned}
$$

By density we may assume that $\left(x_{t}\right)_{t>0} \in \ell_{\infty}\left(S_{\mathcal{M}}\right)$. Now, given $\lambda>0$ and $T>0$, by definition, there exists a projection $e_{T} \in \mathcal{M}$ such that

$$
\left\|e_{T} x_{t} e_{T}\right\|_{\infty} \leq \lambda
$$

for any $t \in(0, T]$, and the right hand side dominates modulo a constant

$$
\lambda \tau\left(1-e_{T}\right)^{\frac{1}{p}} .
$$

Define $u=w^{*}-L_{\infty}-\lim _{\sigma, \mathcal{U}} e_{T}$. Recall that $u$ is not necessarily a projection. However, recalling that $\left(x_{t}\right)_{t>0} \in \ell_{\infty}\left(S_{\mathcal{M}}\right)$, it is straightforward to show that the exact same inequalities above apply for $u$ instead of $e_{T}$, details are left to the reader. Then, the projection $e=\chi_{\left[\frac{1}{2}, 1\right]}(u)$ clearly satisfies

$$
e \leq 2 e u e \leq 4 u^{2} \text { and } 1-e \leq 2(1-u) .
$$

This implies for any $t \in(0, \infty)$ that $\left\|e x_{t} e\right\|_{\infty} \leq 2\left\|y_{t}^{*} u^{2} y_{t}\right\|_{\infty}^{\frac{1}{2}} \leq 2 \lambda$ where $y_{t}$ such that $x_{t}=y_{t}^{*} y_{t}$, and $\lambda \tau(1-e)^{\frac{1}{p}} \leq 2^{\frac{1}{p}} \lambda \tau(1-u)^{\frac{1}{p}}$. Thus we finish the proof. 
Lemma 3.3. Let $1 \leq p \leq \infty$. Then for any $v \in \mathbb{R}^{n}$ and $\left(x_{t}\right)_{t>0}$ in $L_{p}(\mathcal{M})$, we have

and

$$
\left\|\left(x_{t}\right)_{t>0}\right\|_{L_{p}\left(\ell_{\infty}\right)}=\left\|\left(\alpha(v) x_{t}\right)_{t>0}\right\|_{L_{p}\left(\ell_{\infty}\right)}
$$

$$
\left\|\left(x_{t}\right)_{t>0}\right\|_{\Lambda_{p}\left(\ell_{\infty}\right)}=\left\|\left(\alpha(v) x_{t}\right)_{t>0}\right\|_{\Lambda_{p}\left(\ell_{\infty}\right)} .
$$

Proof. We show the first equality. By the definition of $L_{p}\left(\ell_{\infty}\right)$-norm, for any $\varepsilon>0$, there exist a factorization $x_{t}=a y_{t} b$ such that

$$
\varepsilon+\left\|\left(x_{t}\right)_{t>0}\right\|_{L_{p}\left(\ell_{\infty}\right)} \geq\|a\|_{2 p} \sup _{t>0}\left\|y_{t}\right\|_{\infty}\|b\|_{2 p} .
$$

Since $\alpha$ is a homomorphism, we find a factorization of $\alpha(v) x_{t}$,

$$
\alpha(v) x_{t}=(\alpha(v) a)\left(\alpha(v) y_{t}\right)(\alpha(v) b)
$$

with

$$
\|\alpha(v) a\|_{2 p} \sup _{t>0}\left\|\alpha(v) y_{t}\right\|_{\infty}\|\alpha(v) b\|_{2 p}=\|a\|_{2 p} \sup _{t>0}\left\|y_{t}\right\|_{\infty}\|b\|_{2 p} .
$$

Since $\varepsilon$ is arbitrary, we obtain

$$
\left\|\left(x_{t}\right)_{t>0}\right\|_{L_{p}\left(\ell_{\infty}\right)} \geq\left\|\left(\alpha(v) x_{t}\right)_{t>0}\right\|_{L_{p}\left(\ell_{\infty}\right)} .
$$

The reverse inequality is shown similarly.

The second equality follows from the following observation

$$
\begin{aligned}
& \inf _{e \in \mathcal{P}(\mathcal{M})}\left\{\left(\tau\left(e^{\perp}\right)\right)^{\frac{1}{p}}:\left\|e \alpha(v) x_{t} e\right\|_{\infty} \leq \lambda\right\} \\
& =\inf _{e \in \mathcal{P}(\mathcal{M})}\left\{\left(\tau\left(\left(\alpha\left(v^{-1}\right) e\right)^{\perp}\right)\right)^{\frac{1}{p}}:\left\|\alpha(v)\left(\alpha\left(v^{-1}\right)(e) x_{t} \alpha\left(v^{-1}\right)(e)\right)\right\|_{\infty} \leq \lambda\right\} \\
& =\inf _{\alpha\left(v^{-1}\right) e \in \mathcal{P}(\mathcal{M})}\left\{\left(\tau\left(\left(\alpha\left(v^{-1}\right) e\right)^{\perp}\right)\right)^{\frac{1}{p}}: \|\left(\alpha\left(v^{-1}\right)(e) x_{t} \alpha\left(v^{-1}\right)(e) \|_{\infty} \leq \lambda\right\} .\right.
\end{aligned}
$$

where we have used the facts $\alpha\left(v^{-1}\right) e^{\perp}=\left(\alpha\left(v^{-1}\right) e\right)^{\perp}$ and $\alpha$ is a tracepreserving homomorphism.

Lemma 3.4. Let $1 \leq p \leq \infty$ and $\left(f_{t}\right)_{t>0} \subset L_{p}\left(L_{\infty}\left(\mathbb{R}^{n}\right) \bar{\otimes} \mathcal{M}\right)$. Then we have

$$
\left(\int_{\mathbb{R}^{n}}\left\|\left(f_{t}(v)\right)_{t>0}\right\|_{L_{p}\left(\mathcal{M} ; \ell_{\infty}\right)}^{p} d v\right)^{\frac{1}{p}} \leq\left\|\left(f_{t}\right)_{t>0}\right\|_{L_{p}\left(L_{\infty}\left(\mathbb{R}^{n}\right) \bar{\otimes} \mathcal{M} ; \ell_{\infty}\right)}
$$

and

$$
\begin{aligned}
\int_{\mathbb{R}^{n}} & \inf _{e_{v} \in \mathcal{P}(\mathcal{M})}\left\{\tau\left(e_{v}^{\perp}\right):\left\|e_{v} f_{t}(v) e_{v}\right\|_{\infty} \leq \lambda\right\} d v \\
\leq & \inf _{e \in \mathcal{P}\left(L_{\infty}\left(\mathbb{R}^{n}\right) \bar{\otimes} \mathcal{M}\right)}\left\{\tau \int\left(e^{\perp}\right):\left\|e f_{t} e\right\|_{\infty} \leq \lambda\right\} .
\end{aligned}
$$


Proof. By definition, for any $\varepsilon>0$, there exists a factorization $f_{t}=a g_{t} b$ such that

$$
\|a\|_{2 p} \sup _{t}\left\|g_{t}\right\|_{\infty}\|b\|_{2 p} \leq\left\|\left(f_{t}\right)_{t>0}\right\|_{L_{p}\left(\ell_{\infty}\right)}+\varepsilon .
$$

We see that for each $v \in \mathbb{R}^{n}$, we find a factorization $f_{t}(v)=a(v) g_{t}(v) b(v)$ such that the left hand side is smaller than

$$
\left(\int_{\mathbb{R}^{n}}\|a(v)\|_{2 p}^{p} \sup _{t}\left\|g_{t}(v)\right\|_{\infty}^{p}\|b(v)\|_{2 p}^{p} d v\right)^{\frac{1}{p}} \leq\|a\|_{2 p} \sup _{t}\left\|g_{t}\right\|_{\infty}\|b\|_{2 p} .
$$

Since $\varepsilon$ is arbitrary, we obtain the desired result.

Now we show the second inequality. For any $\varepsilon>0$, there exists a projection $e_{\varepsilon}$ such that

$$
\tau \int\left(e_{\varepsilon}^{\perp}\right) \leq \inf _{e \in \mathcal{P}\left(L_{\infty}\left(\mathbb{R}^{n}\right) \bar{\otimes} \mathcal{M}\right)}\left\{\tau \int\left(e^{\perp}\right):\left\|e f_{t} e\right\|_{\infty} \leq \lambda\right\}+\varepsilon .
$$

Observing that for each $v \in \mathbb{R}^{n}$, we find a projection $e_{\varepsilon}(v)$ such that

$$
\left\|e_{\varepsilon}(v) f_{t}(v) e_{\varepsilon}(v)\right\|_{\infty} \leq \lambda,
$$

so the left hand side is controlled by

$$
\int_{\mathbb{R}^{n}} \tau\left(e_{\varepsilon}(v)^{\perp}\right) d v
$$

which yields the desired estimate since $\varepsilon$ is arbitrary.

Now we are at a position to prove Theorem 3.1.

Proof. The case of the strong type $(p, p)$. By Lemma 3.2, it suffices to prove for any $T>0$

$$
\left\|\left(\alpha\left(\nu_{t}\right) x\right)_{0<t \leq T}\right\|_{L_{p}\left(\ell_{\infty}\right)}^{p} \leq C_{p}^{p}\|x\|_{p}^{p}
$$

for any $x \in L_{p}(\mathcal{M})$ where $C_{p}$ is a constant independent of $x$ and $T$.

Given any $S>0$, by Lemma 3.3 and Lemma 3.4, the left hand side of (3.1) equals

$$
\begin{aligned}
& \frac{1}{\left|B_{S}\right|} \int_{B_{S}}\left\|\left(\alpha(v) \alpha\left(\nu_{t}\right) x\right)_{0<t \leq T}\right\|_{L_{p}\left(\mathcal{M} ; \ell_{\infty}\right)}^{p} d v \\
& \quad \leq \frac{1}{\left|B_{S}\right|}\left\|\left(\chi_{B_{S}}(v) \alpha(v) \alpha\left(\nu_{t}\right) x\right)_{0<t \leq T}\right\|_{L_{p}\left(L_{\infty}\left(\mathbb{R}^{n}\right) \bar{\otimes} \mathcal{M} ; \ell_{\infty}\right)}^{p} .
\end{aligned}
$$

Define the $L_{p}(\mathcal{M})$-valued function $f$ on $\mathbb{R}^{n}$ by $f(u)=\chi_{|u| \leq S+C T}(u) \alpha(u) x$. Then for $0<t \leq T$, it is easy to check

$$
\chi_{B_{S}}(v) \alpha(v) \alpha\left(\nu_{t}\right) x=\chi_{B_{S}}(v) \lambda \bar{\otimes} i d_{\mathcal{M}}\left(\nu_{t}\right) f(v) .
$$


Thus the left hand side of (3.1) is not bigger than

$$
\frac{1}{\left|B_{S}\right|}\left\|\left(\chi_{B_{S}}(v) \lambda \bar{\otimes} i d_{\mathcal{M}}\left(\nu_{t}\right) f(v)\right)_{0<t \leq T}\right\|_{L_{p}\left(L_{\infty}\left(\mathbb{R}^{n}\right) \bar{\otimes} \mathcal{M} ; \ell_{\infty}\right)}^{p}
$$

which is smaller than

$$
\frac{1}{\left|B_{S}\right|}\left\|\left(\lambda \bar{\otimes} i d_{\mathcal{M}}\left(\nu_{t}\right) f(v)\right)_{0<t \leq T}\right\|_{L_{p}\left(L_{\infty}\left(\mathbb{R}^{n}\right) \bar{\otimes} \mathcal{M} ; \ell_{\infty}\right)}^{p}
$$

by restricting to $B_{S}$ the functions appearing in the factorization of the latter norm. Then by the assumption, we finish the proof

$$
\leq \frac{1}{\left|B_{S}\right|} C_{p}^{p}\|f\|_{p}^{p}=\frac{\left|B_{S+C T}\right|}{\left|B_{S}\right|} C_{p}^{p}\|x\|_{p}^{p} \rightarrow C_{p}^{p}\|x\|_{p}^{p}
$$

as $S \rightarrow \infty$.

The case of the weak type $(p, p)$ : By the fact that every $x \in L_{p}(\mathcal{M})$ can be written as linear combination of four positive elements, and Lemma 3.2, it suffices to prove for any $\lambda>0$ and $T>0$

$$
\inf _{e \in \mathcal{P}(\mathcal{M})}\left\{\tau\left(e^{\perp}\right):\left\|e \alpha\left(\nu_{t}\right) x e\right\|_{\infty} \leq \lambda, \forall 0<t \leq T\right\} \leq C_{p}^{p} \frac{\|x\|_{p}^{p}}{\lambda^{p}} .
$$

for any $x \in L_{p}^{+}(\mathcal{M})$ where $C_{p}$ is a constant independent of $x$ and $T$. Now the arguments in the proof of estimate (3.1) works well for estimate (3.2) using Lemma 3.3 and 3.4 .

Remark 3.5. From the proof, it is easy to observe that if we replace $\left(\nu_{t}\right)_{t>0}$ by a sequence of integrable functions $\left(\varphi_{t}\right)_{t>0}$ with compact supports in Theorem 3.1, the same conclusion holds.

\section{Noncommutative Wiener's ergodic theorem}

In this section, we show a noncommutative version of Wiener's ergodic theorem. The main result is stated as follows.

Theorem 4.1. The sequence of normalized Lebesgue measures $\left(\beta_{r}\right)_{r>0}$ on the balls in $\mathbb{R}^{n}$ is a both global and local noncommutative pointwise ergodic family for all $1 \leq p<\infty$.

As in the classical setting, we use density argument to show the pointwise convergence. We need the following noncommutative Wiener's ergodic maximal inequality. Let $\left(\mathcal{M}, \tau, \mathbb{R}^{n}, \alpha\right)$ be a fixed $W^{*}$-dynamical system.

Theorem 4.2. Let $x \in L_{1}(\mathcal{M})$, then there exists a constant $C_{n}$ probably depending on $n$ such that

$$
\left\|\left(\alpha\left(\beta_{r}\right) x\right)_{r>0}\right\|_{\Lambda_{1}\left(\ell_{\infty}\right)} \leq C_{n}\|x\|_{1} .
$$


That is, for any $\lambda>0$, there exists a projection $e \in \mathcal{M}$ such that

$$
\left\|e \alpha\left(\beta_{r}\right)(x) e\right\|_{\infty} \leq \lambda, \forall r>0 \text { and } \tau\left(e^{\perp}\right) \leq \frac{C_{n}\|x\|_{1}}{\lambda} .
$$

Let $x \in L_{p}(\mathcal{M})$ with $1<p \leq \infty$, then there exists a constant $C_{p, n}$ probably depending on $n$ such that

$$
\left\|\left(\alpha\left(\beta_{r}\right) x\right)_{r>0}\right\|_{L_{p}\left(\ell_{\infty}\right)} \leq C_{p, n}\|x\|_{p} .
$$

These maximal ergodic inequalities follow from the transference principleTheorem 3.1 and the operator-valued Hardy-Littlewood maximal inequalities. Note that the noncommutative version of Hardy-Littlewood maximal inequality was firstly shown by Mei in [23], his approach is based on noncommutative Doob's inequality, yielding the $O\left(2^{n}\right)$ order of the constants $C_{n}$ and $C_{p, n}$. It is worth to mention that $C_{n}$ can be at most of order $O(n)$ and $C_{p, n}$ can be taken to be independent of $n$ if we appeal to the dimension free estimates established in [12], which are based on Junge/Xu's noncommutative DunfordSchwartz maximal ergodic theorem [18] and Stein's original arguments [29]. This type of dimension free estimates in the noncommutative setting should be interesting in its own right that we will not discuss in the present paper.

Let us finish the proof of Theorem 4.1.

Proof. (i) The global case. We begin with the definition of a dense subspace. Define $S=\left\{x-\alpha(v) x: x \in L_{1}(\mathcal{M}) \cap L_{\infty}(\mathcal{M}), v \in \mathbb{R}^{n}\right\}$. We claim that $S$ is dense in $\overline{(I-F) L_{p}(\mathcal{M})}$ for all $1 \leq p<\infty$. It suffices to verify the claim in the case $p=2$, since it is well-known that $L_{p}(\mathcal{M}) \cap L_{2}(\mathcal{M})$ is dense in $L_{p}(\mathcal{M})$ for any $1 \leq p<\infty$. We only need to prove that $\langle x, z\rangle=0 \forall x \in S$ implies $z \in F\left(L_{2}(\mathcal{M})\right)$. Take $x=y-\alpha(v) y$, clearly we have

$$
0=\langle x, z\rangle=\langle y, z-\alpha(v) z\rangle
$$

which implies $z=F(z)$ since $y \in L_{1}(\mathcal{M}) \cap L_{\infty}(\mathcal{M})$ and $v \in \mathbb{R}^{n}$ are arbitrary.

Next we show the following fact: For any $x \in S$ and any $1 \leq p \leq \infty$, we have

$$
\left\|\alpha\left(\beta_{r}\right) x\right\|_{p} \rightarrow 0, \text { as } r \rightarrow \infty .
$$

Take $x=y-\alpha(v) y \in S$, then

$$
\begin{aligned}
\alpha\left(\beta_{r}\right) x & =\frac{1}{\left|B_{r}\right|} \int_{B_{r}} \alpha(w) y d w-\frac{1}{\left|B_{r}\right|} \int_{v+B_{r}} \alpha(w) y d w \\
& =\int_{\mathbb{R}^{n}} \frac{\chi_{B_{r}}(w)-\chi_{v+B_{r}}(w)}{\left|B_{r}\right|} \alpha(w) y d w .
\end{aligned}
$$


Hence by Minkowski inequality

$$
\begin{aligned}
\left\|\alpha\left(\beta_{r}\right) x\right\|_{p} & \leq \int_{\mathbb{R}^{n}} \frac{\left|\chi_{B_{r}}(w)-\chi_{v+B_{r}}(w)\right|}{\left|B_{r}\right|}\|\alpha(w) y\|_{p} d w \\
& \leq \frac{\left|B_{r} \Delta\left(v+B_{r}\right)\right|}{\left|B_{r}\right|}\|y\|_{p} \rightarrow 0 \text { as } r \rightarrow \infty .
\end{aligned}
$$

Then (4.3) immediately yields the mean ergodic theorem in $L_{p}$ for $1 \leq p<\infty$, that is, $\left\|\alpha\left(\beta_{r}\right) x-F(x)\right\|_{p} \rightarrow 0$ for all $x \in L_{p}(\mathcal{M})$.

Now let us show that $\left(\beta_{r}\right)_{r>0}$ is a global noncommutative pointwise ergodic family in $L_{1}$. That is, for $x \in L_{1}(\mathcal{M})$ and fixed $\varepsilon>0$, we want to find a projection $e$ such that $\tau\left(e^{\perp}\right) \leq \varepsilon$ and $\left\|e\left(\alpha\left(\beta_{r}\right) x-F(x)\right) e\right\|_{\infty} \rightarrow 0$, as $r \rightarrow \infty$. First of all, by the fact $S$ is dense in $\overline{(I-F) L_{1}(\mathcal{M})}$, for any $\delta>0$ we can find $y \in S$ such that $\|x-F(x)-y\|_{1} \leq \delta$. Now apply Theorem 4.2 to the operator $x-F(x)-y$, for any $\lambda>0$, there exists a projection $p$ such that

$$
\tau\left(p^{\perp}\right) \leq \frac{C \delta}{\lambda}, \text { and }\left\|p\left(\alpha\left(\beta_{r}\right)(x-F(x)-y)\right) p\right\|_{\infty} \leq \lambda .
$$

Take $e=p$ and $\lambda=C \delta / \varepsilon$, we have $\tau\left(e^{\perp}\right) \leq \varepsilon$ and

$$
\begin{aligned}
\left\|e\left(\alpha\left(\beta_{r}\right) x-F(x)\right) e\right\|_{\infty} & \leq\left\|e\left(\alpha\left(\beta_{r}\right)(x-F(x)-y)\right) e\right\|_{\infty}+\left\|e\left(\alpha\left(\beta_{r}\right) y\right) e\right\|_{\infty} \\
& \leq C \delta / \varepsilon+\left\|e\left(\alpha\left(\beta_{r}\right) y\right) e\right\|_{\infty},
\end{aligned}
$$

which tends to 0 as $r$ tends to $\infty$ due to (4.3) and the fact that $\delta$ can be taken as small as desired.

Fix $1<p<\infty$, by Remark 2.2, it suffices to prove $\left(\alpha\left(\beta_{r}\right)(x)-F(x)\right)_{r>0} \in$ $L_{p}\left(\mathcal{M} ; c_{0}\right)$ for all $x \in L_{p}(\mathcal{M})$. By the fact that $L_{p}\left(\mathcal{M} ; c_{0}\right)$ is Banach space, $S$ is dense in

$$
\overline{(I-F) L_{p}(\mathcal{M})}
$$

and the maximal inequality (4.2), we are reduced to prove $\left(\alpha\left(\beta_{r}\right) x\right)_{r>0} \in$ $L_{p}\left(\mathcal{M} ; c_{0}\right)$ for all $x \in S$. Fix $x=y-\alpha(v) y \in S$, choose $1<q<p$. Let $0<s<t$, by (4.2), we have

$$
\begin{aligned}
\left\|\sup _{s<r<t}{ }^{+} \alpha\left(\beta_{r}\right) x\right\|_{p} & \leq\left\|\sup _{s<r<t}{ }^{+} \alpha\left(\beta_{r}\right) x\right\|_{q}^{q / p}\left(\sup _{s<r<t}\left\|\alpha\left(\beta_{r}\right) x\right\|_{\infty}\right)^{1-q / p} \\
& \leq\left(C_{q}\|x\|_{q}\right)^{q / p}\left(\sup _{s<r<t} \frac{\left|B_{r} \Delta\left(v+B_{r}\right)\right|}{\left|B_{r}\right|}\|y\|_{\infty}\right)^{1-q / p},
\end{aligned}
$$

which tends to 0 as $s$ tends to $\infty$. Thus $\left(\alpha\left(\beta_{r}\right) x\right)_{r>0}$ is approximated by $\left(\alpha\left(\beta_{r}\right) x\right)_{0<r<s}$ 's in $L_{p}\left(\mathcal{M} ; \ell_{\infty}\right)$, whence belongs to $L_{p}\left(\mathcal{M} ; c_{0}\right)$.

(ii) The local case. The dense subspace we consider in this case is the following one

$$
\mathcal{D}=\left\{\alpha(\phi)(x): \phi \in C_{c}^{\infty}\left(\mathbb{R}^{n}\right), x \in L_{1}(\mathcal{M}) \cap L_{\infty}(\mathcal{M})\right\} .
$$

The density is trivial from the fact that the action $\alpha$ is strong continuous in $L_{p}$ for $1 \leq p<\infty$. The fact $\left(\beta_{r}\right)_{r>0}$ is mean ergodic (as $r \rightarrow 0$ ) in $L_{p}$ for 
all $1 \leq p<\infty$ can also be deduced from the strong continuity of $\alpha$. But we prefer to give a more precise estimate for $x \in \mathcal{D}$. Fix $x=\alpha(\phi)(y) \in \mathcal{D}$, we have

$$
\begin{aligned}
\left\|\alpha\left(\beta_{r}\right)(x)-x\right\|_{p} & =\left\|\alpha\left(\beta_{r}\right) \alpha(\phi)(y)-\alpha(\phi)(y)\right\|_{p} \\
& \leq\left\|\beta_{r} * \phi-\phi\right\|_{1}\|y\|_{p} \leq C_{\phi} r\|y\|_{p},
\end{aligned}
$$

from which we can also easily deduce the mean ergodicity of $\left(\beta_{r}\right)_{r>0}$. The point we will use is that estimate (4.4) is also true when $p=\infty$.

Now the fact that $\left(\beta_{r}\right)_{r>0}$ is a local noncommutative pointwise ergodic family for all $1 \leq p<\infty$ can be shown using the similar arguments having appeared in the global case, by considering $\mathcal{D}$ (resp. (4.4)) instead of $S$ (resp. $(4.3))$.

\section{Noncommutative Jones' ERGOdic theorem}

In this section, we show a noncommutative version of Jones' ergodic theorem. The main result is stated as follows.

Theorem 5.1. The sequence of normalized Lebesgue measures $\left(\sigma_{r}\right)_{r>0}$ on the spheres in $\mathbb{R}^{n}$ is a both global and local noncommutative pointwise ergodic family for all $n /(n-1)<p<\infty, n \geq 3$.

As in the proof of Theorem 4.1, this pointwise ergodic theorem would be established using density argument if we could show the maximal inequality and could find a dense subset on which the pointwise convergence holds. While the maximal inequality now follows easily from the noncommutative transference principle - Theorem 3.1 and the operator-valued version of Stein's spherical maximal inequality-Proposition 4.1 of [12], a dense subset is difficult to find since a priori the dense subset $S$ contructed in the proof of Theorem 4.1 is not a good candidate for Jones' ergodic theorem due to the fact the sphere measures are singular.

One way to find a dense subset is via spectral method as done in the commutative setting [25]. However as we can see in the Appendix that noncommutative analogue of this approach only works for $n \geq 4$. Below we present a method which covers the case $n=3$ based on transference principle inspired by the observation in the commutative setting [14].

We need the following lemma. Fix a $W^{*}$-dynamical system $\left(\mathcal{M}, \tau, \mathbb{R}^{n}, \alpha\right)$.

Lemma 5.2. Let $p>1$. Let $\varphi$ be a radial smooth compactly supported function on $\mathbb{R}^{n}$. For $r>0$, we define $\varphi_{r}(u)=\frac{1}{r^{n}} \varphi\left(\frac{u}{r}\right)$. Then for any $x \in L_{p}(\mathcal{M})$, $\alpha\left(\varphi_{r}\right)$ x converges to $\int \varphi \cdot F(x)$ (resp. $\left.\int \varphi \cdot x\right)$ b.a.u. as $r \rightarrow \infty($ resp. $r \rightarrow 0)$.

Proof. We only show the global case. By Lemma 2.2, it suffices to show $\left(\alpha\left(\varphi_{r}\right) x-\int \varphi \cdot F(x)\right)_{r} \in L_{p}\left(\mathcal{M} ; c_{0}\right)$. Since $\varphi$ is radial, we write $\varphi(u)=\varphi_{0}(|u|)$. 
Using the fact that $\varphi$ is compactly supported, by polar decomposition and integration by parts, we have

$$
\alpha\left(\varphi_{r}\right) x-\int \varphi \cdot F(x)=-|B(0,1)| \int_{0}^{\infty} \varphi_{0}^{\prime}(s) s^{n}\left(\alpha\left(\beta_{s r}\right) x-F(x)\right) d s,
$$

where $\varphi_{0}^{\prime}$ is the derivative of $\varphi_{0}$. Now we clearly obtain the desired result by Wiener's ergodic theorem-Theorem 4.1 and the fact that $L_{p}\left(\mathcal{M} ; c_{0}\right)$ is a Banach space since $\int_{0}^{\infty}\left|\varphi_{0}^{\prime}(s)\right| s^{n} d s<\infty$.

Let $\psi_{0}$ be the Fourier transform of a radial $C_{c}^{\infty}\left(\mathbb{R}^{n}\right)$ function. Assume further that $\psi_{0}(0)=1$ and that, for $1<j<n / 2$,

$$
\partial_{r}^{j} \psi_{0}=0
$$

where $\partial$ is the radial derivation operator. Such a function can be constructed in the following way: Let $\psi$ be any function that is the Fourier transform of a radial $C_{c}^{\infty}\left(\mathbb{R}^{n}\right)$ function and such that $\psi(0)=1$. For $\xi \in S^{n-1}$ and $r \geq 0$, we then define

$$
\psi_{0}(r \xi)=\left(\sum_{j=0}^{n} a_{j} r^{2 j}\right) \psi(r \xi)
$$

where the $a_{j}$ 's are chosen inductively so as to have $\psi_{0}(0)=1$ and then the required number of derivatives to vanish at 0 .

Let us now define for $j \geq 1, \psi_{j}(\xi)=\psi_{0}\left(\xi / 2^{j}\right)-\psi_{0}\left(\xi / 2^{j-1}\right)$. Note that $\psi_{j}$ is still the Fourier transform of a radial compactly supported function, and for every $\xi \in \mathbb{R}^{n}$,

$$
\sum_{j=0}^{\infty} \psi_{j}(\xi)=1
$$

For $j \geq 0$, let $m_{j}:=\hat{\sigma} \psi_{j}$ and $\sigma_{j, 1}=\hat{m}_{j}$. Here $\sigma$ is the normalized Lebesgue measure on the unit sphere $S^{n-1}$ Define $\sigma_{j, r}(u)=\frac{1}{r^{n}} \sigma_{j, 1}\left(\frac{u}{r}\right)$. Using these notations, it is easy to check that for any $r>0$, we have $\sigma_{r}=\sum_{j=0}^{\infty} \sigma_{j, r}$ in the distribution sense. The following estimate plays the key role in showing Theorem 5.1.

Proposition 5.3. Let $1<p \leq 2$, and $n \geq 3$. For each $j \geq 0$ and $f \in$ $L_{p}\left(\mathbb{R}^{n} ; L_{p}(\mathcal{M})\right)$,

$$
\left\|\left(\sigma_{j, r} * f\right)_{r>0}\right\|_{L_{p}\left(\ell_{\infty}\right)} \leq C 2^{(n / p-(n-1)) j}\|f\|_{p} .
$$

We put off its proof after we show Theorem 5.1.

Proof. Interpolating estimate (5.1) with the trivial estimate in $p=\infty$, we have for any $n /(n-1)<p<\infty$, there exists a $Q_{p}>0$ such that

$$
\left\|\left(\sigma_{j, r} * f\right)_{r>0}\right\|_{L_{p}\left(\ell_{\infty}\right)} \leq C 2^{-Q_{p} j}\|f\|_{p},
$$


for any $f \in L_{p}\left(\mathbb{R}^{n} ; L_{p}(\mathcal{M})\right)$. Since $\sigma_{j, 1}$ has compact support, applying the transference principle-Remark 3.5, we have for $x \in L_{p}(\mathcal{M})$,

$$
\left\|\left(\alpha\left(\sigma_{j, r}\right) x\right)_{r>0}\right\|_{L_{p}\left(\ell_{\infty}\right)} \leq C 2^{-Q_{p} j}\|x\|_{p}
$$

Fix one $x \in L_{p}(\mathcal{M})$. From this estimate, we see that $\sum_{j=0}^{J} \alpha\left(\sigma_{j, r}\right) x$ is uniformly convergent in $L_{p}(\mathcal{M})$, which allows us to identify $\alpha\left(\sigma_{r}\right) x$ as its limit. On the other hand, from Lemma 5.2, for all $J \in \mathbb{N}$

$$
\left(\alpha\left(\sum_{j=0}^{J} \sigma_{j, r}\right) x-\int \sum_{j=0}^{J} \sigma_{j} \cdot F(x)\right)_{r>0} \in L_{p}\left(\mathcal{M} ; c_{0}\right) .
$$

Observe that

$$
\begin{aligned}
& \left\|\left(\alpha\left(\sigma_{r}\right) x-F(x)\right)_{r>0}-\left(\alpha\left(\sum_{j=0}^{J} \sigma_{j, r}\right) x-\int \sum_{j=0}^{J} \sigma_{j} \cdot F(x)\right)_{r>0}\right\|_{L_{p}\left(\ell_{\infty}\right)} \\
& \leq\left\|\left(\alpha\left(\sigma_{r}\right) x-\alpha\left(\sum_{j=0}^{J} \sigma_{j, r}\right) x\right)_{r>0}\right\|_{L_{p}\left(\ell_{\infty}\right)}+\left\|\left(1-\int \sum_{j=0}^{J} \sigma_{j}\right) \cdot F(x)\right\|_{p} \\
& \leq C \sum_{j=J+1}^{\infty}\left\|\left(\alpha\left(\sigma_{j, r}\right) x\right)_{r>0}\right\|_{L_{p}\left(\ell_{\infty}\right)}+\left\|\left(1-\int \sum_{j=0}^{J} \sigma_{j}\right) \cdot F(x)\right\|_{p} \\
& \leq C \sum_{j=J+1}^{\infty} 2^{-Q_{p} j}\|x\|_{p}+\left\|\left(1-\int \sum_{j=0}^{J} \sigma_{j}\right) \cdot F(x)\right\|_{p},
\end{aligned}
$$

which tends vers 0 , as $J \rightarrow \infty$. Therefore we have $\left(\alpha\left(\sigma_{r}\right) x-F(x)\right)_{r>0} \in$ $L_{p}\left(\mathcal{M} ; c_{0}\right)$ due to the fact that $L_{p}\left(\mathcal{M} ; c_{0}\right)$ is complete. Thus by Lemma 2.2 we show that $\left(\sigma_{r}\right)_{r>0}$ is a global noncommutative pointwise ergodic family for all $n /(n-1)<p<\infty, n \geq 3$.

The assertion that $\left(\sigma_{r}\right)_{r>0}$ is a local noncommutative pointwise ergodic family for all $n /(n-1)<p<\infty, n \geq 3$ can be shown similarly, just by replacing $F(x)$ by $x$ in the previous arguments.

Now let us show Proposition 5.3. We will show estimate (5.1) by establishing the end-point estimates $p=1,2$ and then using noncommutative version of Marcinkiewicz interpolation theorem. It is worth to mention that contrary to the at most one-page proof of classical Marcinkiewicz interpolation theorem, the proof of noncommutative analogue is quite delicate. It was first shown in [18], then was improved or further generalized in [3] [7]. Using the noncommuative Marcinkiewicz interpolation theorem, we are reduced to show the end-point estimates.

Lemma 5.4. For all $j \geq 0$ and $\left.f \in L_{1}\left(\left(\mathbb{R}^{n}\right) \bar{\otimes} \mathcal{M}\right)\right)$, we have

$$
\left\|\sup _{r}^{+} \sigma_{j, r} * f\right\|_{1, \infty} \leq C 2^{j}\|f\|_{1} \text {. }
$$


As in the commutative setting, this estimate follows essentially from the operator-valued Hardy-Littlewood maximal inequality and the fact that for any $M>n$, there exists $C_{M}<\infty$ such that

$$
\left|\sigma_{j}(u)\right| \leq C_{M} 2^{j}(1+|u|)^{-M}
$$

It is worth to mention that due to noncommutativity, elementary inequalities such as $|x+y| \leq|x|+|y|$ do not hold, so we can not just copy the classical arguments. See for instance Theorem 4.3 of [5].

Lemma 5.5. For any $j \geq 0$ and $\left.f \in L_{2}\left(\left(\mathbb{R}^{n}\right) \bar{\otimes} \mathcal{M}\right)\right)$, we have

$$
\left\|\sup _{r>0}^{+} \sigma_{j, r} * f\right\|_{2} \leq C 2^{(1 / 2-(n-1) / 2) j}\|f\|_{2} \text {. }
$$

Proof. For $j=0$, the method in showing Lemma 5.4 applies. We only need to show $j \geq 1$. By density, it suffices to show the desired estimate for $S_{\mathcal{M}}^{+}$-valued Schwartz function $f$. We consider the following two column $g$-functions:

$$
G_{j}(f)(u)=\left(\int_{0}^{\infty}\left|\sigma_{j, t} * f(u)\right|^{2} \frac{d t}{t}\right)^{\frac{1}{2}}
$$

and

$$
\tilde{G}_{j}(f)(u)=\left(\int_{0}^{\infty}\left|\tilde{\sigma}_{j, t} * f(u)\right|^{2} \frac{d t}{t}\right)^{\frac{1}{2}}
$$

where

$$
\tilde{\sigma}_{j, t}=t \frac{d \sigma_{j, t}}{d t}
$$

By the fundamental theorem of calculus, we deduce that

$$
\begin{aligned}
& \left(\sigma_{j, t} * f\right)^{2}=\int_{\varepsilon}^{t} \frac{d}{d s}\left(\sigma_{j, s} * f\right)^{2} d s+\left(\sigma_{j, \varepsilon} * f\right)^{2} \\
& =\int_{\varepsilon}^{t} \tilde{\sigma}_{j, s} * f^{*} \sigma_{j, s} * f+\sigma_{j, s} * f^{*} \tilde{\sigma}_{j, s} * f \frac{d s}{s}+\left(\sigma_{j, \varepsilon} * f\right)^{2} \\
& \leq \int_{\varepsilon}^{t}\left|\tilde{\sigma}_{j, s} * f^{*} \sigma_{j, s} * f+\sigma_{j, s} * f^{*} \tilde{\sigma}_{j, s} * f\right| \frac{d s}{s}+\left(\sigma_{j, \varepsilon} * f\right)^{2} .
\end{aligned}
$$


Hence by triangle inequality and Hölder inequality, we have

$$
\begin{aligned}
\left\|\sup _{t}\left|\sigma_{j, s} * f\right|^{2}\right\|_{1}^{1 / 2} \leq & \left\|\int_{0}^{\infty}\left|\tilde{\sigma}_{j, s} * f^{*} \sigma_{j, s} * f+\sigma_{j, s} * f^{*} \tilde{\sigma}_{j, s} * f\right| \frac{d s}{s}\right\|_{1}^{1 / 2} \\
& +\left\|\left(\sigma_{j, \varepsilon} * f\right)^{2}\right\|_{1}^{1 / 2} \\
\leq & 2\left\|\int_{0}^{\infty} \tilde{\sigma}_{j, s} * f^{*} \sigma_{j, s} * f\right\|_{1}^{1 / 2} \\
& +2\left\|\int_{0}^{\infty} \sigma_{j, s} * f^{*} \tilde{\sigma}_{j, s} * f \frac{d s}{s}\right\|_{1}^{1 / 2}+\left\|\left(\sigma_{j, \varepsilon} * f\right)^{2}\right\|_{1}^{1 / 2} \\
\leq & 4\left\|G_{j}(f)\right\|_{2}^{\frac{1}{2}}\left\|\tilde{G}_{j}(f)\right\|_{2}^{\frac{1}{2}}+\left\|f_{\varepsilon, j}(x)^{2}\right\|_{1}^{1 / 2} . \\
\leq & 8\left\|G_{j}(f)\right\|_{2}^{\frac{1}{2}}\left\|\tilde{G}_{j}(f)\right\|_{2}^{\frac{1}{2}} .
\end{aligned}
$$

The last inequality is due to the fact that $\left\|f_{\varepsilon, j}(x)^{2}\right\|_{1}^{1 / 2}$ tends to 0 as $\varepsilon$ tends to $\infty$ by Lebesgue dominated theorem. The rest of the arguments are similar to those in classical setting [26] or [14]. That is, based on the estimates

$$
|\hat{\sigma}(\xi)|+|\nabla \hat{\sigma}(\xi)| \leq C_{n}(1+|\xi|)^{(1-n) / 2},
$$

using Plancherel's theorem and the properties of $\psi_{j}$ to finish the proof. We omit the details.

\section{ApPEndix}

In this Appendix, we present the spectral method to find a dense subset in order to prove noncommutative pointwise ergodic theorems, which might be useful in other cases when the transference principle is not available, for instance, when the underlying group is not amenable.

To prove the pointwise ergodic theorem, we first need the following maximal ergodic inequality, which follows from the noncommutative transference principle-Theorem 3.1 and the operator-valued version of Stein's spherical maximal inequality-Proposition 4.1 of [12]. Let $\left(\mathcal{M}, \tau, \mathbb{R}^{n}, \alpha\right)$ be a fixed $W^{*}$-dynamical system.

Theorem 6.1. Let $n \geq 3$ and $p>n /(n-1)$. Let $x \in L_{p}(\mathcal{M})$. Then there exists a constant $C_{p, n}$ such that

$$
\left\|\sup _{r>0}^{+} \alpha\left(\sigma_{r}\right) x\right\|_{p} \leq C_{p, n}\|x\|_{p} .
$$

Let $K_{n}:=S O(n)$ denote the rotation group. The Euclidean motion group $G_{n}:=\mathbb{R}^{n} \rtimes K_{n}$ with group law of $G_{n}$ given by

$$
\left(u_{1}, k_{1}\right)\left(u_{2}, k_{2}\right)=\left(u_{1}+k_{1} u_{2}, k_{1} k_{2}\right) .
$$


The inverse of an element $(u, k) \in G_{n}$ is given by $\left(-u, k^{-1}\right)$ and $(0, I)$ serves as the identity $e$ where $I$ is the $n \times n$ identity matrix. Then $K_{n}$ and $\mathbb{R}^{n}$ are isomorphic to two subgroups of $G_{n}$.

A function $f$ on $\mathbb{R}^{n}$ is said to be radial if $f$ is a function of $|u|$, equivalently $f(k u)=f(u)$ for all $u \in \mathbb{R}^{n}$ and $k \in K_{n}$. Let $L^{1}\left(\mathbb{R}^{n}, K_{n}\right)$ denote the subspace of radial functions in $L^{1}\left(\mathbb{R}^{n}\right)$. This space is canonically identical with $L^{1}\left(G_{n}, K_{n}\right)$, the subspace of bi- $K_{n}$-invariant functions in $L^{1}\left(G_{n}\right)$. The radial functions on $\mathbb{R}^{n}$ form a commutative convolution algebra, since this algebra is canonically isomorphic to the algebra of bi- $K_{n}$-invariant functions on $G_{n}$ and it is well-known (see e.g. [8], [28]) that $\left(G_{n}, K_{n}\right)$ form a Gelfand pair.

As it is well-known (see e.g. [4]), the complex homomorphisms of a Gelfand pair $L^{1}\left(G_{n}, K_{n}\right)$ are given by bounded spherical functions. Bounded $K_{n^{-}}$ spherical functions on $G_{n}$ are characterized by satisfying $\phi(e)=1$ and the integrals equation

$$
\int_{K_{n}} \phi(a k b) d k=\phi(a) \phi(b), a, b \in G_{n} .
$$

The family of spherical functions is given for each $s>0$ by

$$
\varphi_{s}(u)=\frac{2^{\frac{n-2}{2}} \Gamma\left(\frac{n}{2}\right)}{(s|u|)^{\frac{n-2}{2}}} J_{\frac{n-2}{2}}(s|u|)
$$

where $J_{\frac{n-2}{2}}$ is the Bessel function of order $\frac{n-2}{2}$, and for $s=0$, the spherical function $\varphi_{0}(u)=1$ identically. Thus the Gelfand spectrum $\Sigma$ of the algebra $L^{1}\left(G_{n}, K_{n}\right)$, equivalently the algebra $L^{1}\left(\mathbb{R}^{n}, K_{n}\right)$, is the union of the Bessel spectrum $(0, \infty)$ and the trivial character $\{0\}$. In what follows $\varphi_{s}(r)$ stands for $\varphi_{s}(u)$ with $|u|=r$ when $s>0$ and $\varphi_{s}(r)=1$ identically when $s=0$.

Let $M\left(\mathbb{R}^{n}, K_{n}\right)$ denote the norm-closed convolution algebra generated by the surface measures $\left\{\sigma_{r}\right\}_{r>0}$ in $M\left(\mathbb{R}^{n}\right)$. Since finite linear combinations of functions of the form $\sigma_{r_{1}} * \sigma_{r_{2}} * \cdots * \sigma_{r_{k}}$ where $r_{i}>0$ and $k \geq 3$ are dense in $L^{1}\left(\mathbb{R}^{n}, K_{n}\right)$ whenever $n \geq 3$, and thus $L^{1}\left(\mathbb{R}^{n}, K_{n}\right) \subset M\left(\mathbb{R}^{n}, K_{n}\right)$ as a subalgebra. Let $\psi$ be a non-zero continuous complex homomorphism of $M\left(\mathbb{R}^{n}, K_{n}\right)$. Then the radial function $\varphi(g)=\psi\left(m_{K_{n}} * \delta_{g} * m_{K_{n}}\right)$ equals $\varphi_{s}(g)$ for some $s \in \Sigma$. Whence, restriction of complex characters from $M\left(\mathbb{R}^{n}, K_{n}\right)$ to its subalgebra $L^{1}\left(\mathbb{R}^{n}, K_{n}\right)$ induces a canonical identification of the Gelfand spectrum of the two algebras.

The previous explanations enable us to do the following arguments. Being a weak $*$-continuous action of $\mathbb{R}^{n}$ on a von Neumann algebra $\mathcal{M}, \alpha$ induces a strongly continuous unitary representation of $\mathbb{R}^{n}$ on $L_{2}(\mathcal{M})$. Then $\alpha$ determines canonically a norm continuous $*$-representation of the algebra $M\left(\mathbb{R}^{n}, K_{n}\right)$. Let us denote by $\mathcal{A}_{\alpha}$ the commutative $C^{*}$-algebra which is the closure of $\alpha\left(M\left(\mathbb{R}^{n}, K_{n}\right)\right)$ in the operator norm. Let $\Sigma_{\alpha}$ denote the spectrum of $\mathcal{A}_{\alpha}$ which is by definition the set of all non-zero norm continuous complex homomorphisms of $\mathcal{A}_{\alpha}$. Clearly $\Sigma_{\alpha}$ is a subset of the Gelfand spectrum 
of $M\left(\mathbb{R}^{n}, K_{n}\right)$. Consequently, every symmetric (self-adjoint) measure $\mu$ in $M\left(\mathbb{R}^{n}, K_{n}\right)$ is mapped to a self-adjoint operator on $L_{2}(\mathcal{M})$, whose spectrum is the set $\left\{\varphi_{s}(\mu): s \in \Sigma_{\alpha}\right\}$. As a consequence, we have the following spectral decomposition,

$$
\alpha\left(\sigma_{r}\right)=\int_{s \in \Sigma_{\alpha}} \varphi_{s}(r) d e_{s} .
$$

Moreover, we need the following asymptotic estimates of spherical functions.

Lemma 6.2. Fix $\varepsilon>0$, and let $\Sigma_{\varepsilon}=\left\{s: \varepsilon \leq s \leq \varepsilon^{-1}\right\}$. Then

$$
\sup _{s \in \Sigma_{\varepsilon}}\left|\varphi_{s}(r)\right| \leq C_{\varepsilon, n}(1+r)^{-\frac{n-1}{2}},
$$

where $C_{\varepsilon, n}$ is a positive constant independent of $r$.

The estimates follow from the standard expansion of the Bessel functions at infinity. See for instance Appendix B.7 of [9] for detailed informations.

Theorem 6.3. Let $n \geq 4$. Let $x \in L_{p}(\mathcal{M})$. The family $\alpha\left(\sigma_{r}\right) x$ converges to $F(x)$, b.a.u for $n /(n-1)<p<\infty$ as $r \rightarrow \infty$.

Proof. Let us first prove the case $p=2$. Note that $L_{2}(\mathcal{M})$ is the orthogonal sum of three closed subspace: $\mathcal{H}_{1}=$ the space of operators invariant under each $\alpha\left(\sigma_{r}\right), \mathcal{H}_{\Sigma}=$ the space of operators whose spectral measure is supported in the Bessel spectrum, and finally, $\mathcal{H}_{0}=$ the space of operators in the kernel of each $\alpha\left(\sigma_{r}\right)$. Clearly, in the first and the third subspace we have $\alpha\left(\sigma_{r}\right) x-$ $F(x)=0$. Hence by Lemma 2.2, it suffices to prove $\left(\alpha\left(\sigma_{r}\right) x\right)_{r>1} \in L_{2}\left(\mathcal{M} ; c_{0}\right)$ for $x \in \mathcal{H}_{\Sigma}$. By the maximal ergodic theorem-Theorem 6.1, it suffices to prove $\left(\alpha\left(\sigma_{r}\right) x\right)_{r>1} \in L_{2}\left(\mathcal{M} ; c_{0}\right)$ for $x$ in some dense set of $\mathcal{H}_{\Sigma}$. Indeed, suppose we have $\left(\alpha\left(\sigma_{r}\right) y\right)_{r>1} \in L_{2}\left(\mathcal{M} ; c_{0}\right)$ for all $y$ in a dense set of $\mathcal{H}_{\Sigma}$. Then for fixed $x \in \mathcal{H}_{\Sigma}$, for any $\delta>0$, there exists a $y$ in the dense subset of $\mathcal{H}_{\Sigma}$, such that $\|x-y\|_{2} \leq \delta$. Therefore by maximal inequality (6.1)

$$
\left\|\left(\alpha\left(\sigma_{r}\right) x\right)_{r>1}-\left(\alpha\left(\sigma_{r}\right) y\right)_{r>1}\right\|_{L_{2}\left(\ell_{\infty}\right)} \leq C\|x-y\|_{2} \leq C \delta .
$$

Since $\delta$ is arbitrary, $\left(\alpha\left(\sigma_{r}\right) x\right)_{r>1}$ is in the closure of $L_{2}\left(\mathcal{M} ; c_{0}\right)$, thus belongs to $L_{2}\left(\mathcal{M} ; c_{0}\right)$ because $L_{2}\left(\mathcal{M} ; c_{0}\right)$ is closed.

For $\varepsilon>0$, let $\mathcal{H}_{\varepsilon}$ be the subspace of operators $x \in L_{2}(\mathcal{M})$ whose spectral measure $\langle\operatorname{de}(x), x\rangle$ is supported in $\Sigma_{\varepsilon}$ defined in Lemma 6.2. The dense set of $\mathcal{H}_{\Sigma}$ we shall consider is $\mathcal{H}_{\varepsilon}$. For $x \in \mathcal{H}_{\varepsilon}$, by spectral decomposition (6.2) as well as the spectral estimates (6.3), we have

$$
\left\|\alpha\left(\sigma_{r}\right) x\right\|_{2} \leq \sup _{s \in \Sigma_{\varepsilon}}\left|\varphi_{s}(r)\right|\|x\|_{2} \leq C_{\varepsilon, n} r^{-\frac{n-1}{2}}\|x\|_{2} .
$$


Then the fact that the space $L_{2}\left(\mathcal{M} ; c_{0}\right)$ is complete yields that $\left(\alpha\left(\sigma_{r}\right) x\right)_{r>1} \in$ $L_{2}\left(\mathcal{M} ; c_{0}\right)$, since

$$
\left(\alpha\left(\sigma_{r}\right) x\right)_{r>1}=\int_{1}^{\infty}\left(\alpha\left(\sigma_{r}\right) x\right)_{r=t} d t
$$

and

$$
\int_{1}^{\infty} t^{-n / 2+1 / 2} d t<\infty
$$

For other cases $p \neq 2$, using again the maximal ergodic theorem-Theorem 6.1, it suffices to prove $\left(\alpha\left(\sigma_{r}\right) x\right)_{r>1} \in L_{p}\left(\mathcal{M} ; c_{0}\right)$ for $x \in L_{1}(\mathcal{M}) \cap \mathcal{M}$, which is a dense subset of $L_{p}(\mathcal{M})$. Without loss of generality, we assume $p<2$. Find $q$ and $\theta$ such that $(2 n-1) /(2 n-2)<q<p$ and $1 / p=(1-\theta) / q+\theta / 2$. Let $x \in L_{1}(\mathcal{M}) \cap \mathcal{M}$. For any $1<t<s$, by maximal inequality (6.1),

$$
\begin{aligned}
\left\|\left(\alpha\left(\sigma_{r}\right) x\right)_{t<r<s}\right\|_{L_{p}\left(\ell_{\infty}\right)} & \leq\left\|\left(\alpha\left(\sigma_{r}\right) x\right)_{t<r<s}\right\|_{L_{q}\left(\ell_{\infty}\right)}^{1-\theta}\left\|\left(\alpha\left(\sigma_{r}\right) x\right)_{t<r<s}\right\|_{L_{2}\left(\ell_{\infty}\right)}^{\theta} \\
& \leq C_{q}^{1-\theta}\|x\|_{q}\left\|\left(\alpha\left(\sigma_{r}\right) x\right)_{t<r<s}\right\|_{L_{2}\left(\ell_{\infty}\right)}^{\theta},
\end{aligned}
$$

which tends to 0 when $t$ tends to $\infty$ using the result in the case $p=2$. Hence $\left(\alpha\left(\sigma_{r}\right) x\right)_{r>1}$ is approximated by $\left(\alpha\left(\sigma_{r}\right) x\right)_{1<r<t}$ 's, therefore belongs to $L_{p}\left(\mathcal{M} ; c_{0}\right)$.

Remark 6.4. The reason why the spectral method only works for $n \geq 4$ is due to the fact that estimate (6.4) is not true for $n \leq 3$.

Acknowledgement. The work is partially supported by the NSF of China11601396, Funds for Talents of China-413100002 and 1000 Young Talent Researcher Programm of China-429900018-101150.

\section{REFERENCES}

[1] C. Anantharaman-Delaroche, On ergodic theorems for free group actions on noncommutative spaces, Probab. Theory Related Fields, 135 (4): 520-546, 2006.

[2] T. Bekjan, Noncommutative maximal ergodic theorems for positive contractions, J. Funct. Anal. 254(9): 24012418, 2008.

[3] T. Bekjan, Z. Chen and A. Osekowski, Noncommutative maximal inequalities associated with convex functions, Trans. Amer. Math. Soc. 369: 409-427, 2017.

[4] C. Benson, J. Jenkins and G. Ratcliff, Bounded $K$-spherical functions on Heisenberg groups. J. Funct. Anal. 105: 409-443, 1992.

[5] Z. Chen, Z. Yin and Q. Xu, Harmonic analysis on quantum tori, Comm. Math. Phys. 322(3): 755805, 2013.

[6] A. Defant and M. Junge, Maximal theorems of Menchoff-Rademacher type in noncommutative $L_{q}$-spaces, J.Funct.Anal. 206: 322-355, 2004.

[7] S. Dirksen, Weak-type interpolation for noncommutative maximal operators, J. Operator Theory 73(2): 515532, 2015.

[8] J. Faraut, K. Harzallah, Deux cours d'analyse harmonique, Birkhäuser Verlag, Basel, 1987.

[9] L. Grafakos, Classical Fourier Analysis (Graduate Texts in Mathematics), Springer; 2nd ed. edition, 2008. 
[10] G. Hong, Noncommutative maximal ergodic theorems for spherical means on the Heisenberg group, arXiv:1611.01651 .

[11] G. Hong, M. Sun, Noncommutative multi-parameter Wiener-Wintner type ergodic theorem, arXiv:1602.00927.

[12] G. Hong, The behavior of the bounds of matrix-valued maximal inequality in $\mathbb{R}^{n}$ for large $n$, Illinois. J. Math., 57(3): 855-869, 2013.

[13] Y. Hu, Maximal ergodic theorems for some group actions, Journal of Functional Analysis, 254: 1282-1306, 2008.

[14] P. Jaming, The spherical ergodic theorem revisited, Expositiones Mathematicae, 27(3): 257-269, 2009.

[15] M. Junge, Doob's inequality for non-commutative martingales, J. Reine Angew. Math., 549: 149-190, 2002.

[16] M. Junge, T. Mei and J. Parcet, Smooth Fourier multipliers on group von Neumann algebras, preprint 2011, arXiv Math: 1010.5320.

[17] M. Junge, J. Parcet, Mixed-norm inequalities and operator space Lp embedding theory, Mem. Amer. Math. Soc. 952, 2010.

[18] M. Junge and Q. Xu, Noncommutative maximal ergodic theorems, J. Amer. Math. Soc. 20: 385-439, 2006.

[19] R. Jones, Ergodic averages on spheres, J. Anal. Math, 61: 29-45, 1993.

[20] E. C. Lance, Ergodic theorems for convex sets and operator algebras, Invent. Math., 37(3): 201-214, 1976.

[21] M. T. Lacey, Ergodic averages on circles, J. Anal. Math. 67: 199-206, 1995.

[22] S. Litvinov: A non-commutative Winener-Wintner theorem, arXiv:1405.4427v1, math.OA (2014), 1-10.

[23] T. Mei, Operator valued Hardy spaces, Mem. Amer. Math. Soc. 188, 2007.

[24] A. Nevo, Pointwise Ergodic Theorems for Actions of Groups, Chapter 13 of Handbook of Dynamical Systems, Vol 1, Part B, 871982, 2006.

[25] A. Nevo and S. Thangavelu, Pointwise ergodic theorems for radial averages on the Heisenberg Group, Adv. Math., 127: 307-334, 1997.

[26] J. L. Rubio de Francia, Maximal functions and Fourier transforms, Duke Math. J., 53: 395-404, 1986.

[27] E.M. Stein, J.O. Stromberg, Behavior of maximal functions in $\mathbb{R}^{n}$ for large n, Arkiv Math. 21: 259-269, 1983.

[28] R. Strichartz, $L^{p}$ harmonic analysis and Radon transform on the Heisenberg group, J. Funct. Anal. 96: 350-406, 1991.

[29] E.M. Stein, J.O. Strömberg, Behavior of maximal functions in $\mathbb{R}^{n}$ for large $n$, Arkiv Math. 21: 259-269, 1983

[30] G. Pisier, Non-commutative vector-valued $L_{p}$-space and completely $p$-summing maps, Astérisque, 247, 1998.

[31] G. Pisier and Q. Xu, Non-commutative $L_{p}$-spaces, In Handbook of the Geometry of Banach Spaces, Vol. II, 1459-1517. Edited by W.B. Johnson and J. Lindenstrauss, Elsevier, 2003.

[32] N. Wiener, The ergodic theorem, Duke Math. J., 5: 1-18, 1939.

[33] F.J. Yeadon, Ergodic theorems for semifinite von Neumann algebras I, J. London. Math. Soc. (2), 16(2): 326-332, 1977.

School of Mathematics and Statistics, Wuhan University, Wuhan 430072 China,

E-mail address: guixiang.hong@whu.edu.cn 